\title{
SYMMETRIES IN NUCLEAR STRUCTURE
}


Series Editor: A. Zichichi, European Physical Society, Geneva, Switzerland

Series Editorial Board: P. G. Bergmann, J. Collinge, V. Hughes, N. Kurti, T. D. Lee, K. M. B. Siegbahn, G. 't Hooft, P. Toubert, E. Velikhov, G. Veneziano, G. Zhou

1. Perspectives for New Detectors in Future Supercolliders, 1991

2. Data Structures for Particle Physics Experiments: Evolution or Revolution?, 1991

3. Image Processing for Future High-Energy Physics Detectors, 1992

4. GaAs Detectors and Electronics for High-Energy Physics, 1992

5. Supercolliders and Superdetectors, 1993

6. Properties of SUSY Particles, 1993

7. From Superstrings to Supergravity, 1994

8. Probing the Nuclear Paradigm with Heavy Ion Reactions, 1994

9. Quantum-Like Models and Coherent Effects, 1995

10. Quantum Gravity, 1996

11. Crystalline Beams and Related Issues, 1996

12. The Spin Structure of the Nucleon, 1997

13. Hadron Colliders at the Highest Energy and Luminosity, 1998

14. Universality Features in Multihadron Production and the Leading Effect, 1998

15. Exotic Nuclei, 1998

16. Spin in Gravity: Is It Possible to Give an Experimental Basis to Torsion?, 1998

17. New Detectors, 1999

18. Classical and Quantum Nonlocality, 2000

19. Silicides: Fundamentals and Applications, 2000

20. Superconducting Materials for High Energy Colliders, 2001

21. Deep Inelastic Scattering, 2001

22. Electromagnetic Probes of Fundamental Physics, 2003

23. Epioptics-7, 2004

24. Symmetries in Nuclear Structure, 2004 


\title{
THE SCIENCE AND CULTURE SERIES - PHYSICS
}

Proceedings of the Highly Specialized Seminar on

\section{SYMMETRIES IN \\ NUCLEAR STRUCTURE}

\author{
An Occasion to Celebrate the \\ 60th Birthday of Francesco lachello
}

Erice, Italy $\quad 23-30$ March 2003

Editors

\section{Andrea Vitturi and Richard F. Casten}

Series Editor

A. Zichichi 


\section{Published by}

World Scientific Publishing Co. Pte. Ltd.

5 Toh Tuck Link, Singapore 596224

USA office: Suite 202, 1060 Main Street, River Edge, NJ 07661

UK office: 57 Shelton Street, Covent Garden, London WC2H 9HE

\section{British Library Cataloguing-in-Publication Data}

A catalogue record for this book is available from the British Library.

\section{Cover Illustrations}

Front: Roman mosaic ca $200 \mathrm{BC}$

Back: Seventeenth century map of Sicily by Dutch cartographer Jansson

\section{SYMMETRIES IN NUCLEAR STRUCTURE: AN OCCASION TO CELEBRATE THE 60TH BIRTHDAY OF FRANCESCO IACHELLO}

Copyright $(\mathcal{C} 2004$ by World Scientific Publishing Co. Pte. Ltd.

All rights reserved. This book, or parts thereof, may not be reproduced in any form or by any means, electronic or mechanical, including photocopying, recording or any information storage and retrieval system now known or to be invented, without written permission from the Publisher.

For photocopying of material in this volume, please pay a copying fee through the Copyright Clearance Center, Inc., 222 Rosewood Drive, Danvers, MA 01923, USA. In this case permission to photocopy is not required from the publisher.

ISBN $981-238-812-5$ 


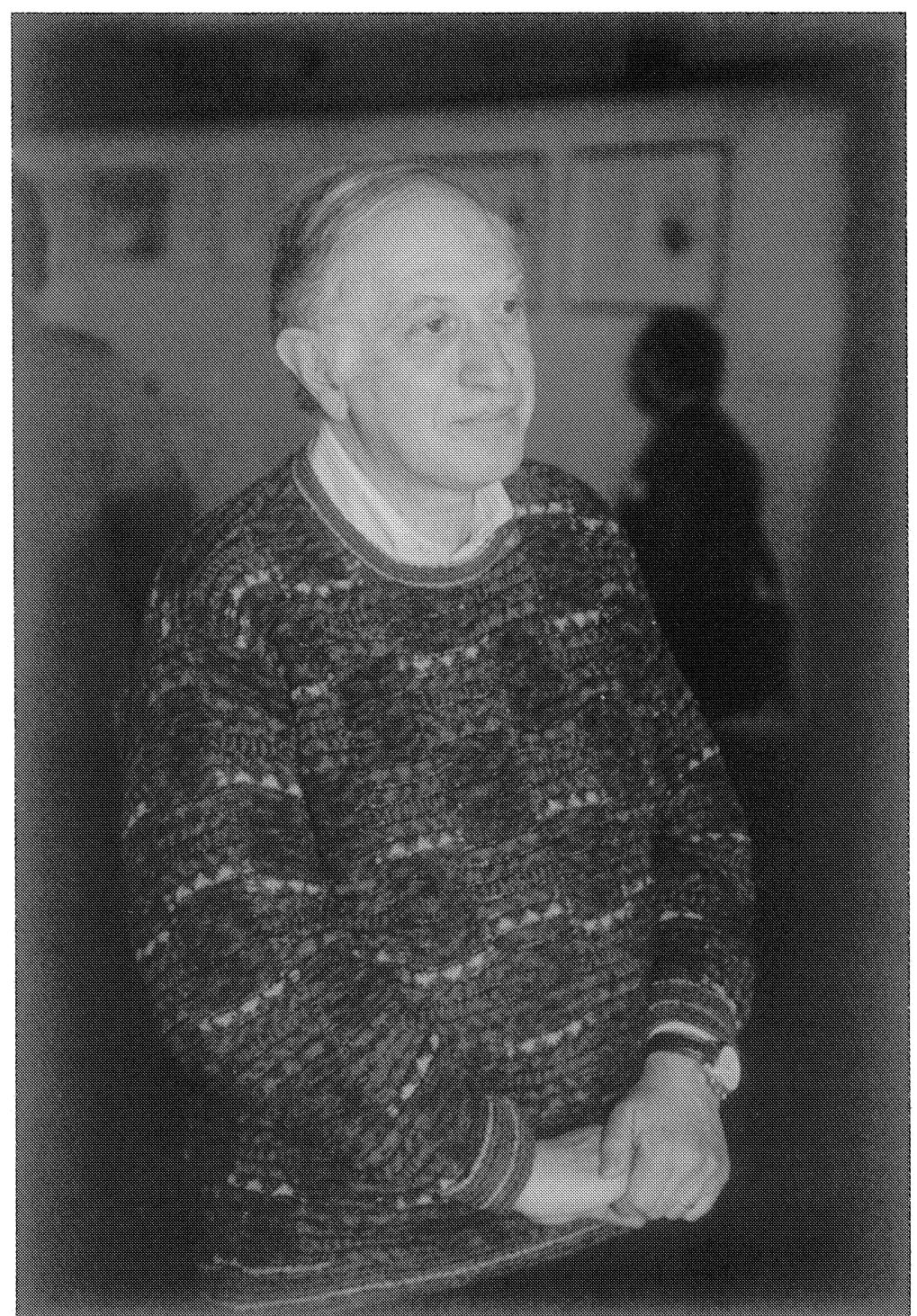

Professor Francesco Iachello 
This page is intentionally left blank 


\section{CONTENTS}

Preface

A. Vitturi, R. F. Casten, editors

Symmetry: the search for order in nature

F. Iachello

Additional quantum numbers, dynamical symmetries and degeneracies

I. Talmi

Partial dynamical symmetry as an intermediate symmetry structure

A. Leviatan

Vector coherent state theory: a powerful tool for solving algebraic problems in physics

D. J. Rowe

Symmetries in strongly deformed nuclei

J.P. Draayer, G. Popa, J. G. Hirsch, C. E. Vargas

Shape coexistence and its symmetries

$\underline{K}$. Heyde, R. Fossion

Shape-invariance and many-body physics

A. B. Balantekin

Some new perspectives on pairing in nuclei

S. Pittel. J. Dukelsky

Pairing and quartetting in the Interacting Boson Model

$P$. Van Isacker

Challenges from symmetry on the drip lines

D. D. Warner 
High accuracy atomic mass measurements. Application to neutron-rich zirconium isotopes

J. Äystö, V. Kolhinen, S. Rinta-Antila, S. Kopecky, J. Hakala, J. Huikari, A. Jokinen, A. Nieminen, J. Szerypo

Nuclear supersymmetry: new tests and extensions

A. Frank, J. Barea, R. Bijker

Everything you always wanted to know about SUSY, but were afraid to ask

R. Bijker, J. Barea, A. Frank

Supersymmetry and identical bands

P. von Brentano

Searching for boson-fermion symmetries in neutron-rich nuclei

J. C. Blackmon, C. J. Gross, F. Liang, D. Shapira, M. S. Smith,

D. W. Stracener, R. L. Kozub, C. D. Nesaraja, U. Greife,

R. J. Livesay, Z. Ma

Supersymmetry in nuclear clusterization

G. Lévai

Cluster effects in alternating parity and superdeformed bands of medium mass and heavy nuclei

G. G. Adamian, N. V. Antonenko, R. V. Jolos, Yu. V. Palchikov,

W. Scheid, T. M. Shneidman

Thermal signatures of phase transitions in finite nuclei

Y. Alhassid

Integrability and quantum phase transitions in Interacting Boson Models 
Critical point nuclei in the Interacting Boson Model

N. V. Zamfir, E. A. McCutchan, R. F. Casten

Critical points in nuclei and Interacting Boson Model intrinsic states

J. N. Ginocchio, A. Leviatan

The critical point symmetry $\mathrm{E}(5)$ and the IBM

J. M. Arias, C. E. Alonso, A. Vitturi, J. E. García-Ramos,

J. Dukelsky, A. Frank

Finite well solutions for the $\mathrm{E}(5)$ and $\mathrm{X}(5)$ Hamiltonians

M. A. Caprio

Analytical solutions of Bohr collective Hamiltonian with $\gamma$-instability

L. Fortunato, A. Vitturi

Phase transitions and critical points in the Interacting Boson Model

J. E. García-Ramos, J. M. Arias, J. Barea, A. Frank

Test of the empirical realization of the $X(5)$ symmetry in ${ }^{150} \mathrm{Nd}$ and ${ }^{104} \mathrm{Mo}$

R. Krücken

The rich structures of a very simple Hamiltonian

J. Jolie

The excited $0^{+}$states in ${ }^{162} \mathrm{Yb}$ and the critical point phase/shape transition

E. A. McCutchan, N. V. Zamfir, R. F. Casten

Test of the critical point symmetry $\mathrm{X}(5)$ in $\mathrm{N}=90$ nuclei and $\mathrm{A} \approx 180$ Os isotopes

A. Dewald, O. Möller, D. Tonev, A. Fitzler, B. Saha, K. Jessen,

S. Heinze, A. Linnemann, J. Jolie, K. O. Zell, P. von Brentano, P. Petkov, R. F. Casten, M. Caprio, N. V. Zamfir, R. Krücken, D. Bazzacco, S. Lunardi, C. Rossi-Alvarez, F. Brandolini, C. Ur, G. de Angelis, D. R. Napoli, E. Farnea, N. Marginean, T. Martinez, M. Axiotis 
Phase transitions in the octupole degree of freedom

P. G. Bizzeti

Prompt particle decay in nuclei: present status and future perspectives C. Fahlander, D. Rudolph

Quadrupole moments and deformations of "shears" states in the $Z=82 \mathrm{~Pb}$ nuclei

D. Balabanski, G. Neyens, K. Vyvey

Structure of bands in neutron-rich even palladium isotopes A. Giannatiempo, A. Nannini, P. Sona

Octupole two-phonon states in deformed nuclei

G. Graw Y. Eisermann, R. Hertenberger, H.-F. Wirth, S. Christen, O. Möller, D. Tonev, J. Jolie, C. Günther, A. I. Levon, N. V. Zamfir

IBFM2 study of odd- $A$ Cs and Xe isotopes and beta decay in Xe-Cs N. Yoshida, L. Zuffi, S. Brant

Algebraic description of high angular momentum states in nuclei 309 D. Vretenar, S. Brant, G. Bonsignori

Search for seniority isomers: lifetime measurements in ${ }^{93} \mathrm{Tc}$ and ${ }^{95} \mathrm{Ru}$ K. P. Lieb E. Galindo, M. Hausmann, A. Jungclaus, I. P. Johnstone, R. Schwengner, A. Dewald, A. Fitzler, O. Möller, G. De Angelis, A. Gadea, T. Martinez, D. R. Napoli, C. Ur

Magnetic moments from the Mediterranean to Mt. Fuji

N. Benczer-Koller, M. J. Taylor, G. Kumbartzki, Y. Y. Sharon,

L. Zamick, T. J. Mertzimekis, A. E. Stuchbery

Probing nuclear structure by real photons: systematics of low-lying dipole modes in heavy nuclei

U. Kneissl

Electric dipole excitations close to the particle threshold

A. Zilges 
Mixed-symmetry multiphonon structures and first evidence for $F$-vector $E 1$ transitions

N. Pietralla, C. Fransen, P. Von Brentano

Spin-isospin excitations, pairing and shape coexistence

E. Moyade Guerra, P. Sarriguren, R. Alvarez-Rodriguez,

A. Escuderos

Dipole symmetry near threshold

M. Gai

Measurement of the spin entanglement of two-proton system

H. Sakai, T. Saito, A. Tamii, T. Kawabata, Y. Satou

SU(6)-breaking symmetry and the ratio of proton momentum distributions

M. M. Giannini, E. Santopinto, A. Vassallo, M. Vanderhaeghen

The hypercentral constituent quark model and its symmetry

M.M. Giannini, E. Santopinto

Manifestation of symmetry properties of nucleon structure in strong and electromagnetic processes

E. Tomasi-Gustafsson, M. P. Rekalo

Regularity and chaos in low-lying $2^{+}$states of even-even nuclei

A. Y. Abul-Magd, H. L. Harney, M. H. Simbel, H. A. Weidenmüller

Shape-phase and order-to-chaos transitions in nuclei

406

G. Maino

Some remarks on the symmetry of the superconducting wavefunction in the cuprates
K. A. Müller

Algebraic description of $n$-alkane molecules

S. Oss 
Algebraic approach to vibrationally highly excited polyatomic molecules

\section{K. Yamanouchi, T. Sako}

Molecular quasilinearity under the prism of dynamical symmetry breaking: a detailed study of the methinophosphide $(H C P) \widetilde{A}-\widetilde{X}$ system

F. Pérez-Bernal, F. Iachello, P. H. Vaccaro, H. Ishikawa, H. Toyosaki, N. Mikami

Algebraic methods for the quantitative interpretation of vibronically-resolved molecular spectra: the structure and dynamics of disulfur monoxide $\left(\mathrm{S}_{2} \mathrm{O}\right)$

P. H. Vaccaro, T. Müller, F. Iachello, P. Pérez-Bernal

Franco, the early days

R. H. Siemssen

Poem

R. F. Casten

List of participants

Photographs 


\section{PREFACE}

These Proceedings contain the papers presented at the Highly Specialized Seminar on "Symmetries in Nuclear Structure", which was held at the Ettore Majorana Centre in Erice, Italy, 23-30 March 2003. The meeting was intended to celebrate, on the occasion of his 60th birthday, the career and the remarkable achievements of Francesco Iachello. Since the development of the Interacting Boson Model in the early 1970s, the ideas of Francesco Iachello have provided a variety of frameworks for understanding collective behaviour in nuclear structure, founded in the concepts of dynamical symmetries and spectrum generating algebras. The original ideas, which were developed for the description of atomic nuclei, have now been successfully extended to cover spectroscopic behaviour in other fields, such as molecular or hadronic spectra. More recently, the suggestion by Iachello of Critical Point Symmetries to treat nuclei in shape/phase transitional regions has opened an exciting new front for both theoreticians and experimentalists.

The talks presented at the meeting covered many of the most active forefront areas of nuclear structure as well as other fields where ideas of symmetries are being explored. Topics in nuclear structure included extensive discussions of dynamical symmetries, critical point symmetries, phase transitions, statistical properties of nuclei, supersymmetry, mixed symmetry states, shears bands, pairing and clustering in nuclei, shape coexistence, exotic nuclei, dipole modes, and astrophysics, among others. In addition, important sessions focused on talks by European Laboratory Directors (or their representatives) outlining future prospects for nuclear structure, and the application of symmetry ideas to molecular phenomena. Finally, a special lecture by Nobel Laureate Alex Mueller, on s and d wave symmetry in superconductors, presented a unique insight into an allied field.

On behalf of the Organizing Committee and of all participants we thank the Ettore Majorana Centre and Professor Antonino Zichichi for providing such an ideal venue for the meeting. The location fitted perfectly into the spirit of the reunion, since precisely in this place so many of the early first successes of the Interacting Boson Model were announced in the late 1970 s - early 1980 s.

Finally, we thank Raffaella Ruggiu and Pino Aceto from the Majorana Centre for the perfect organization in Erice and Annarosa Spalla from INFN, Padova, for the highly professional management of the conference and her fundamental help in the collection and the editing of these proceedings.

Andrea Vitturi and Richard F. Casten 